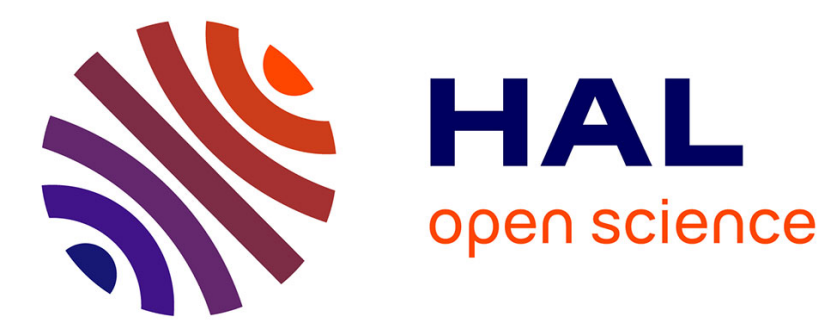

\title{
PHASE TRANSITIONS IN TWO- AND THREE-DIMENSIONAL COMPOUND-LATTICE MODELS
}

H. Neumann

\section{- To cite this version:}

H. Neumann. PHASE TRANSITIONS IN TWO- AND THREE-DIMENSIONAL COMPOUNDLATTICE MODELS. Journal de Physique Colloques, 1977, 38 (C4), pp.C4-133-C4-140. 10.1051/jphyscol:1977421 . jpa-00217137

\section{HAL Id: jpa-00217137 https://hal.science/jpa-00217137}

Submitted on 1 Jan 1977

HAL is a multi-disciplinary open access archive for the deposit and dissemination of scientific research documents, whether they are published or not. The documents may come from teaching and research institutions in France or abroad, or from public or private research centers.
L'archive ouverte pluridisciplinaire HAL, est destinée au dépôt et à la diffusion de documents scientifiques de niveau recherche, publiés ou non, émanant des établissements d'enseignement et de recherche français ou étrangers, des laboratoires publics ou privés. 


\title{
PHASE TRANSITIONS IN TWO- AND THREE-DIMENSIONAL COMPOUND-LATTICE MODELS
}

\author{
H. P. NEUMANN \\ Institut für Physikalische Chemie der Universität \\ Frankfurt/Main, Frankfurt am Main, G.F.R.
}

\begin{abstract}
Combinatorial state equations of generalized two- or three-dimensional compoundlattice models are derived. Analytical expressions are constructed for the pressure-density functions. The approximation allows the computation of phase diagrams of a wide class of liquid and solid one-component and multicomponent systems consisting of spherical nonpolar molecules.
\end{abstract}

Simple conventional compound-lattice models consisting of two or a few more sublattices can describe in a crude approximation one-component phase systems with fluid and one or a few more crystalline phases [1-5], [11-14].

These compound-lattice models will be now extended in the sense that apart from the fluid phases, a greater number of allotropic solid phases can be described. A more refined description of the periodical structure of the one-particle distribution densities in crystalline phases (thermal expansion included) becomes then also possible. A further extension to multicomponent systems with a greater number of polymorphic solid phases seems to be reasonable and will be therefore performed because simple lattice models for fluid mixtures have been very successful during the last years [6]. The main features of this generalized compound-lattice model may be outlined in the following [7].

Consider an arbitrary Bravais lattice of type BT with an unit cell of volume $v$ and $V$ lattice sites as a basic lattice. In this case, a greater number of the unit cells of the basic lattice can be joined to a real elementary cell with volume $V_{\mathrm{E}}^{\mathrm{BT}}$ of an arbitrary given periodical structure of the same type BT, whereby this new elementary cell is a true to scale enlargement of the unit cell of the basic lattice. In a somewhat different interpretation, the basic lattice can be considered as a composition of a great number of parallelly shifted equivalent sublattices with the real elementary cell (with volume $V_{E}^{\mathrm{BT}}$ ) of the given periodic structure as the unit cell. Every real elementary cell contains then a lot of sublattice sites. Each lattice site in the real elementary cell belongs to a different sublattice. Of course, lattice sites on opposite faces of the real elementary cell are only counted once. The lattice sites of the basic lattice which lie in the real elementary cell and belong to different sublattices can then be considered as coordinate points of a coordinate system with an origin in the midst of the real elementary cell and coordinate axes parallel to the three vectors which span up the real elementary cell. As rectangular or oblique-angled coordinates, the Weiss indices $\mathbf{n}=\left(n_{1}, n_{2}, n_{3}\right)$ can then be used. A basic lattice which is built up in this way by sublattices may be called a compound lattice.

We assume now classical particles of $N$ different species without any internal degrees of freedom, moving on an arbitrary compound lattice selected from a complete system of $Z$ possible compound lattices of all different Bravais types BT and volumes $V_{\mathrm{B}}^{\mathrm{BT}}$ of corresponding elementary cells. The interaction may be a two-body spherical potential interaction $-v_{r s}\left(\left|\mathbf{r}_{i}-\mathbf{r}_{j}\right|\right)$ between the particles of species $r, s$ at positions $\mathbf{r}_{i}, \mathbf{r}_{j}$ with an inserted hard core of volume $v$ of the unit cell of the compound lattice. However potentials which are represented by general symmetric functions with $v_{r s}\left(\mathbf{r}_{i}-\mathbf{r}_{j}\right)=v_{r s}\left(\mathbf{r}_{j}-\mathbf{r}_{i}\right)$ can be also admitted in principal. The function $v_{r s}\left(\left|\mathbf{r}_{i}-\mathbf{r}_{j}\right|\right)$ may be an integrable function, composed of a short ranged but strong soft repulsive part and a long ranged weak attractive tail. The magnitude of the hard core (with volume $v$ ) can be chosen so small that the values of the potential function on the edge of the hard-core unit cell of the compound lattice become so large negative that $\exp \left[v_{r s}\left(\left|\mathbf{r}_{i}-\mathbf{r}_{j}\right|\right)\right]$ becomes negligible small, and that the variation of the potential within the range of a diameter of $v$ becomes also small. The 
system of classical particles which are restricted to move on possible representatives of the complete system of compound lattices may be called generalized compound-lattice model. The generalized compound-lattice model is now able to describe in principle apart from fluid structures every possible periodical crystalline structure. One needs only consider homogeneous particle (species $i$ ) number densities $\rho_{i}\left(n, V_{E}^{\mathrm{BT}}\right)$ on the sublattices $n$ of a compound lattice with elementary cell volume $V_{\mathrm{E}}^{\mathrm{BT}}$. If $\rho_{i}\left(n, V_{\mathrm{B}}^{\mathrm{BT}}\right)$ does not vary with $\mathrm{n}$, a fluid structure is described. If $\rho_{i}\left(n, V_{E}^{B T}\right)$ varies with $n$ in the sense that arbitrary ratios $\rho_{i}\left(\mathrm{n}, V_{\mathrm{E}}^{\mathrm{BT}}\right) / \rho_{i}\left(\mathrm{n}, V_{\mathrm{E}}^{\mathrm{BT}}\right)$ remain constant, a completely disordered crystalline mixture is described. If $\rho_{i}\left(\mathbf{n}, V_{\mathrm{E}}^{\mathrm{BT}}\right)$ as well as some (or all) ratios $\rho_{i}\left(\mathbf{n}, V_{\mathrm{E}}^{\mathrm{BT}}\right) / \rho_{i}\left(\mathbf{n}, V_{\mathrm{E}}^{\mathrm{BT}}\right)$ vary with $\mathbf{n}$, an ordered crystalline mixture with super structure is reproduced.

The thermodynamical properties of our generalized compound-lattice model can be calculated by an evaluation of the grand canonical partition function $\Xi$ in combinatorial approximation :

$$
\begin{aligned}
& \Xi \simeq \sum_{\mathrm{BT}} \sum_{V_{\mathrm{E}}^{\mathrm{BT}}} \frac{1}{Z}\left[\sum_{\left\{\rho_{1}\left(\mathrm{n}, V_{\mathrm{E}}^{\mathrm{BT}}\right)\right\}} \cdots \sum_{\left\{\rho_{N}^{\left.\left(\mathrm{n}, V_{E}^{\mathrm{BT}}\right)\right\}}\right.} \times\right. \\
& \times W_{\mathrm{conf}}\left(V_{\mathrm{E}}^{\mathrm{BT}}\right) \exp \left[-\beta V\left\langle\varepsilon\left(V_{\mathrm{E}}^{\mathrm{BT}}\right)\right\rangle\right] \times \\
&\left.\times \exp \left[\beta V \sum_{i=1}^{N}\left(\sum_{\mathbf{n}} g\left(\mathbf{n}, V_{\mathrm{E}}^{\mathrm{BT}}\right) \rho_{i}\left(\mathrm{n}, V_{\mathrm{E}}^{\mathrm{BT}}\right)\right) \mu_{i}\right]\right],
\end{aligned}
$$

where $Z$ is the number of all possible compound lattices for fixed $v, V$ is the total number of lattice sites on the basic lattice, $g\left(n, V_{\mathrm{E}}^{\mathrm{BT}}\right)$ is the sublattice site distribution function that is the ratio of the number of lattice sites on the sublattice $n$ to $V, N$ is the number of different species of particles, $\mu_{i}$ is the chemical potential of the particle species $i, \beta$ is the reciprocal of $k T$ with $k$ the Boltzmann constant, and $T$ the absolute temperature, $W_{\text {conf }}\left(V_{\mathrm{E}}^{\mathrm{BT}}\right)$ is the number of all configurations with fixed sublattice densities $\rho_{i}\left(\mathbf{n}, V_{\mathrm{E}}^{\mathrm{BT}}\right)$, and $\left\langle\varepsilon\left(V_{\mathrm{E}}^{\mathrm{BT}}\right)\right\rangle$ is the ensemble averaged mean configurational energy per lattice site. The sum symbols $\sum_{\left\{\rho_{i}\left(n, V_{E}^{\mathrm{BX}}\right)\right\}}$ are abbreviations for the multiple sums $\sum_{\rho_{i}\left(\mathrm{n}_{1}, V_{\mathrm{E}}^{\mathrm{BT}}\right)=0}^{1} \cdots \sum_{\rho_{i}\left(\mathrm{n}_{\nu}, V_{\mathrm{E}}^{\mathrm{BT}}\right)=0}^{1}$, where $\mathbf{n}_{1}, \ldots, \mathbf{n}_{\nu}$ are all sublattice indices.

The further proceeding consists in a selection of the maximum term in the sum (1) and its insertion in the fundamental formula

$$
p=\frac{1}{\beta V} \ln \Xi
$$

A detailed calculation on the pressure function by means of (1) and (2) requires an approximate evaluation of the combinatorial factor $W_{\text {conf }}\left(V_{\mathrm{E}}^{\mathrm{BT}}\right)$ and the mean configurational energy $\left\langle\varepsilon\left(V_{E}^{\mathrm{BT}}\right)\right\rangle$. In mean-field approximation both expressions can be easily calculated. The result is :

$$
\begin{aligned}
W_{\text {conf }}\left(V_{\mathrm{E}}^{\mathrm{BT}}\right)=\prod_{\mathbf{m}}^{\alpha\left(V_{\mathrm{E}}^{\mathrm{BT}}\right)}\left\{\left[g\left(\mathbf{m}, V_{\mathrm{E}}^{\mathrm{BT}}\right) V\right] ! / \prod_{t=1}^{N}\left[\rho_{i}\left(\mathbf{m}, V_{\mathrm{E}}^{\mathrm{BT}}\right) g\left(\mathbf{m}, V_{\mathrm{E}}^{\mathrm{BT}}\right) V\right] ! \times\right. & \\
& \left.\times\left[\left(1-\sum_{i=1}^{N} \rho_{i}\left(\mathbf{m}, V_{\mathrm{E}}^{\mathrm{BT}}\right)\right) g\left(\mathbf{m}, V_{\mathrm{E}}^{\mathrm{BT}}\right) V\right] !\right\}
\end{aligned}
$$

and

$$
\left\langle\varepsilon\left(V_{\mathrm{E}}^{\mathrm{BT}}\right)\right\rangle=-\frac{1}{2} \sum_{i, j=1}^{N} \sum_{\mathbf{m}}^{\alpha\left(V_{\mathrm{E}}^{\mathrm{B} T}\right)} \sum_{\mathbf{n}}^{\alpha\left(V_{\mathrm{E}}^{\mathrm{B} T}\right)} g\left(\mathbf{m}, V_{\mathrm{E}}^{\mathrm{BT}}\right) w_{i j}\left(\mathbf{m}, \mathbf{n} ; V_{\mathrm{E}}^{\mathrm{BT}}\right) \rho_{i}\left(\mathbf{m}, V_{\mathrm{E}}^{\mathrm{BT}}\right) \rho_{j}\left(\mathbf{n}, V_{\mathrm{E}}^{\mathrm{BT}}\right)
$$

respectively. The product symbol $\prod_{m}^{\alpha\left(V z^{\top}\right)}$ in (3) is an abbreviation for the multiple product $\prod_{m_{1}=-\alpha_{1}}^{\alpha_{1}-1} \prod_{m_{2}=-\alpha_{2}}^{\alpha_{2}-1} \prod_{m_{3}=-\alpha_{3}}^{\alpha_{3}-1}$ The sum symbols $\sum_{m}^{\alpha(V)}$ in (4) are abbreviations for the multiple sums $\sum_{m_{1}=-\alpha_{1}}^{\alpha-1} \sum_{m_{2}=-\alpha_{2}}^{\alpha_{2}-1} \sum_{m_{3}=-\alpha_{3}}^{\alpha_{3}-1} \cdot \alpha_{1}, \alpha_{2}, \alpha_{3}$ are the components of the vector $\alpha$. The interaction functions $w_{i j}\left(\mathbf{m}, \mathbf{n} ; V_{\mathrm{E}}^{\mathrm{BT}}\right)$ in (4) are defined as

$$
w_{i j}\left(\mathbf{m}, \mathbf{n} ; V_{\mathbf{E}}^{\mathrm{BT}}\right)=\sum_{\mathbf{x}}^{\mathrm{n}}(\mathbf{m}) v_{i j}(|\mathbf{y}-\mathbf{x}|)
$$

where $\mathbf{x}$ runs over all lattice sites of sublattice $\mathbf{n}$, while $\mathbf{y}$ on sublattice $\mathbf{m}$ is kept fixed.

The combinatorial approximation of $W_{\text {conf }}\left(V_{E}^{\mathrm{BT}}\right)$ and $\left\langle\varepsilon\left(V_{E}^{\mathrm{BT}}\right)\right\rangle$ can be refined by considering the statistical correlation at least within the frame of an approximate correct distribution of certain groups of subfigures on the lattice which form overlap sequences. The approximation has been originally developed only for lattice models of fluids [8-10] (or solid binary mixtures respectively) and could be extended also to systems with a few fluid and crystalline phases [2-4], [11-14]. The theory may be now extended to our generalized compound-lattice model. Further, the hierarchies of overlap figures 
may be extended and adapted to the functional course of the particle interaction in such a way that an optimal transfer of the statistical correlation produced by the particle interaction to the thermodynamics of the model is guaranteed without an excessive increasing of the mathematical frame work. Simultaneously, the spatial range, where correlation is considered, can be extended. Further, an extension of the range of the hard-core part of the interaction can be admitted. This program can be realized in several steps.

In a first step, the total range of all possible inter-particle distances is divided into $k=1,-, \Omega$ zones. This division may be rather arbitrary, but the width of a zone should be taken comparatively small, if the interaction potential is strongly varying and comparatively large respectively, if the potential is only weakly varying therein. By every bond of any zone a new sublattice of the basic compound lattice with elementary cells which are concerning the geometric structure true to scale enlargements of those of the basic lattice is generated which has this bond as nearest neighbour link. There may be $\tau=1$, ,$- \sigma$ sublattices which may be called correlation lattices. The correlation lattice $\tau=1$ may be identified with the basic lattice. Some of them are completely (not only geometrically) equivalent in the sense that they can be generated from one another by orthogonal maps in the way that a complete identification of sublattice sites is achieved. Such equivalent correlation lattices can be joined to a super correlation lattice. There may be $\rho=1$, $\varphi$ super correlation lattices with groups of $m_{p}$ correlation lattices respectively.

In a second step, one can consider lattice site clusters on the correlation lattices. These lattice site clusters may be built up by $l$ lattice sites which are arranged in a geometric structure $h_{a}$ with a ratio of sublattice sites $h_{\mathrm{b}}$. The partition of the sublattice sites in the cluster for fixed ratio of their numbers may be denoted by $h_{\mathrm{c}}$. It may be further assumed that the lattice site cluster is a member of an overlap sequence of clusters on the correlation lattice $\tau$ with $i=1,-, n_{\tau}$ stages of overlapping (beginning with the basic figures at $i=1$ and ending with single sublattice sites at $i=n_{\tau}$ ), where at each stage $i$ a group of $\omega_{i}$ overlap figures occur. Such a lattice site cluster may be denoted by $l_{\omega_{i}}^{(\tau)} \boldsymbol{h}_{\omega_{i}}^{(\tau)}$, where $\boldsymbol{h}$ represents the triple $\left(h_{\mathrm{a}}, h_{\mathrm{b}}, h_{\mathrm{c}}\right)$. Confining exclusively to lattice site clusters which are members of overlap sequences, then for all correlation lattices (or super correlation lattices) with nearest neighbour links lying in the same zone of the $\Omega$ zones of interaction distances the same overlap sequence of clusters of geometrically equivalent types is chosen. Geometrically equivalent means here that the clusters are generally true to scale enlargements from one another. For each interaction zone $k$ now a set of basic clusters $l_{\omega_{1}}^{\left(\tau_{k}\right)} h_{\omega_{1}}^{\left(\tau_{k}\right)}$ may be chosen which are again members of an overlap sequence. In the first zone $k=1$, where the interaction is generally most varying with inter-particle distance the basic set $\left\{l_{\omega_{1}}^{\left(\tau_{1}\right)} h_{\omega_{1}}^{\left(\tau_{1}\right)}\right\}$ of clusters of the overlap sequence $\left\{l_{\omega_{1}}^{\left(\tau_{k}\right)} h_{\omega_{1}}^{\left(\tau_{k}\right)}\right\}$ is chosen as a basic set of the overlap sequence $\left\{l_{\omega_{i}}^{\left(\tau_{1}\right)} h_{\omega_{i}}^{\left(\tau_{k}\right)}\right\}$. The following sets $\left\{I_{\omega_{1}}^{\left(\tau_{k^{\prime}}\right)} h_{\omega_{k}}^{\left(\tau_{k}\right)}\right\}$ of overlap clusters are adjoined step by step as basic clusters to the other zones $k$ (where the interaction is step by step less varying), generating there new overlap sequences $\left\{l_{\omega_{k}}^{\left(\tau_{k}\right)} h_{\omega_{k}}^{\left(\tau_{k}\right)}\right\}$. In the zone $k=\Omega-1$ finally, only simple links occur, whereby all links are comprised which are not already counted as overlap subclusters in clusters of the corresponding overlap sequences of the interaction zones $k=1,-, \Omega-2$. In the last zone $k=\Omega$ with unlimited interaction distances, only single isolated lattice sites of all different sublattices are chosen simultaneously as basic and overlap clusters. It may be possible that lattice site clusters of different correlation lattices are contained in one another.

In a third step, our basic compound lattice can be decomposed directly or via intermediate steps into lattice site clusters $l_{\omega_{i}}^{(\tau)} h_{\omega_{i}}^{(\tau)}$ which belong to a well defined stage $i$ in the overlap hierarchy. In such disconnected lattices, the range of the direct correlation is restricted to the lattice site clusters. A globally consideration of statistical correlation up to greater distances is achieved by combinatorial ansätze as [12]-[14] and [23]. The disconnected lattices may be called pseudo lattices and may be designated by $p\left(0, l_{1}, l_{2},-, l_{k} ; V_{\mathrm{E}}^{\mathrm{BT}}\right)$. The meaning of this notation is the following: when $l_{i}$ is the greatest $l$-number of the set $\left\{l_{\omega_{i}}^{\left(\tau_{1}\right)} h_{\omega_{i}}^{\left(\tau_{1}\right)}\right\}$ of the overlap clusters belonging to the interaction zone $k=1$ of smallest distances, then

$$
p\left(0, l_{1}, l_{2},-, l_{n} ; V_{\mathrm{E}}^{\mathrm{BT}}\right)
$$

is the disconnected pseudo lattice which is generated from the basic lattice $p\left(0, V_{\mathrm{E}}^{\mathrm{BT}}\right)$ by successive disconnections in the way:

$$
\begin{aligned}
p\left(0, V_{\mathrm{E}}^{\mathrm{BT}}\right) \rightarrow p\left(0, l_{1} ; V_{\mathrm{E}}^{\mathrm{BT}}\right) & \rightarrow p\left(0, l_{1}, l_{2} ; V_{\mathrm{E}}^{\mathrm{BT}}\right) \rightarrow \cdots \\
& \rightarrow p\left(0, l_{1}, l_{2},-, l_{n} ; V_{\mathrm{E}}^{\mathrm{BT}}\right) .
\end{aligned}
$$

The scheme of disconnection can be chosen completely in the sense that all stages $i$ of the overlap sequences are comprised, but it can be chosen also incompletely in the sense that one or more stages $i$ are overleaped. It is clear that there are a lot of possibilities of disconnection paths which must be all found for a given system of overlap sequences of lattice site clusters.

As further important functions, the lattice site cluster distribution numbers

$$
x_{\tau}\left(0, l_{k},-, l_{i} ; l_{\omega_{i}}^{(\tau)} h_{\omega_{i}}^{(\tau)} ; V_{\mathrm{E}}^{\mathrm{BT}}\right)
$$

giving the ratio of the numbers of lattice site clusters $l_{\omega_{1}}^{(\tau)} h_{\omega_{1}}^{(\tau)}$ on the correlation lattice $\tau$ (as a part of the 
pseudo lattice $\left.p\left(0, l_{k},-, l_{i} ; V_{\mathrm{E}}^{\mathrm{BT}}\right)\right)$ to the total number $V$ of lattice sites, must be determined for all the various pseudo lattices. If $a_{\tau \chi}\left(l_{\omega_{1+j}}^{(\tau)} h_{\omega_{t+j}}^{(\tau)} \mid l_{\omega_{i}}^{(\chi)} h_{\omega_{j}}^{(\chi)} ; V_{\mathrm{E}}^{\mathrm{BT}}\right)$ is the number of lattice site clusters $l_{\omega_{i+j}}^{(\tau)} h_{\omega_{i+j}}^{(\tau)}$ which are contained in the cluster $l_{\omega_{i}}^{(x)} h_{\omega_{i}}^{(x)}$, the cluster distribution numbers can be derived successively by the recursion formula

$$
\begin{aligned}
& x_{\tau}\left(0, l_{k},-, l_{i}, l_{i+j} ; l_{\omega_{i+j}}^{(\tau)} h_{\omega_{i+j}}^{(\tau)} ; V_{\mathrm{E}}^{\mathrm{BT}}\right)= \\
& =\sum_{x} \sum_{\omega_{i}} a_{\tau x}\left(l_{\omega_{i+j}}^{(\tau)} h_{\omega_{i+j}}^{(\tau)} \mid l_{\omega_{i}}^{(x)} h_{\omega_{i}}^{(x)} ; V_{\mathrm{E}}^{\mathrm{BT}}\right) \times \\
& \quad \times x_{\chi}\left(0, l_{k},-, l_{i} ; l_{\omega_{i}}^{(x)} h_{\omega_{i}}^{(x)} ; V_{\mathrm{E}}^{\mathrm{BT}}\right)
\end{aligned}
$$

from the $x_{\tau}\left(0, l_{k} ; l_{\omega_{k}}^{(\tau)} h_{\omega_{k}}^{(\tau)} ; V_{\mathrm{E}}^{\mathrm{BT}}\right)$. The disconnection of the basic lattice into pseudo lattices, consisting of completely uncorrelated lattice site clusters, leads to an overcounting of overlap clusters on the pseudo lattices at the cutting pieces in comparison with the original number of these clusters on the basic compound lattice. This overcounting can be canceled by certain correction terms $y_{\tau}\left(0, l_{i} ; l_{\omega_{i}}^{(\tau)} h_{\omega_{i}}^{(\tau)} ; V_{\mathrm{E}}^{\mathrm{BT}}\right)$ which are defined by the linear algebraic system of equations :

$$
\begin{aligned}
& x_{\tau}\left(0, l_{1} ; l_{\omega_{1}}^{(\tau)} h_{\omega_{1}}^{(\tau)} ; V_{\mathrm{E}}^{\mathrm{BT}}\right)=y_{\tau}\left(0, l_{1} ; l_{\omega_{1}}^{(\tau)} h_{\omega_{1}}^{(\tau)} ; V_{\mathrm{E}}^{\mathrm{BT}}\right)+ \\
& +\sum_{\chi} \sum_{\omega_{1}^{\prime}} a_{\tau_{\chi}}\left(l_{\omega_{1}}^{(\tau)} h_{\omega_{1}}^{(\tau)} \mid l_{\omega_{1}}^{(\chi)} h_{\omega_{1}}^{(x)} ; V_{E}^{B T}\right) x_{\chi}\left(0, l_{1} ; l_{\omega_{1}^{\prime}}^{(\chi)} h_{\omega_{1}^{\prime}}^{(\chi)} ; V_{E}^{\mathrm{BT}}\right), \\
& x_{\tau}\left(0, l_{2} ; l_{\omega_{2}}^{(\tau)} h_{\omega_{2}}^{(\tau)} ; V_{\mathrm{E}}^{\mathrm{BT}}\right)=y_{\tau}\left(0, l_{2} ; l_{\omega_{2}}^{(\tau)} h_{\omega_{2}}^{(\tau)} ; V_{\mathrm{E}}^{\mathrm{BT}}\right)+ \\
& +\sum_{x} \sum_{\omega_{1}} a_{\tau x}\left(l_{\omega_{2}}^{(\tau)} h_{\omega_{2}}^{(\tau)} \mid l_{\omega_{1}}^{(x)} h_{\omega_{1}}^{(x)} ; V_{\mathrm{E}}^{\mathrm{BT}}\right) \times \\
& \times\left[y_{\chi}\left(0, l_{1} ; l_{\omega_{1}}^{(x)} h_{\omega_{1}}^{(x)} ; V_{\mathrm{E}}^{\mathrm{BT}}\right)+x_{\chi}\left(0, l_{1} ; l_{\omega_{1}}^{(x)} h_{\omega_{1}}^{(x)} ; V_{\mathrm{E}}^{\mathrm{BT}}\right)\right] \\
& x_{\tau}\left(0, l_{n_{\tau}-1} ; l_{\omega_{n_{\tau}-1}}^{(\tau)} h_{\omega_{n_{\tau}-1}}^{(\tau)} ; V_{\mathrm{E}}^{\mathrm{BT}}\right)=y_{\tau}\left(0, l_{n_{\tau}-1} ; l_{\omega_{n_{\tau}-3}}^{(\tau)} h_{\omega_{n_{\tau}-1}}^{(\tau)} ; V_{\mathrm{E}}^{\mathrm{BT}}\right)+ \\
& +\sum_{x} \sum_{\omega_{n_{\tau}-2}} a_{\tau \chi}\left(l_{\omega_{n_{\tau}-1}}^{(\tau)} h_{\omega_{n_{\tau}-1}}^{(\tau)} \mid l_{\omega_{n_{r}-2}}^{(x)} h_{\omega_{\omega_{\tau}-2}}^{(x)} ; V_{\mathrm{E}}^{\mathrm{BT}}\right) \times \\
& \times y_{x}\left(0, l_{n_{\tau}-2} ; l_{\omega_{n_{\tau}-2}}^{(x)} h_{\omega_{n_{\tau}-2}}^{(x)} ; V_{\mathrm{E}}^{\mathrm{BX}}\right)+ \\
& +\sum_{\varepsilon} \sum_{\omega_{n_{\tau}-3}} a_{\tau \varepsilon}\left(l_{\omega_{n_{\tau}-1}}^{(\tau)} h_{\omega_{n_{\tau}-1}}^{(\tau)} \mid l_{\omega_{n_{\tau}}-3}^{(\varepsilon)} h_{\omega_{n_{\tau}-3}}^{(\varepsilon)} ; V_{E}^{\mathrm{BT}}\right) \times \\
& \times y_{\varepsilon}\left(0, l_{n_{\tau}-3} ; l_{\omega_{n \tau-3}}^{(\varepsilon)} h_{\omega_{n \tau-3}}^{(\varepsilon)} ; V_{\mathrm{E}}^{\mathrm{BT}}\right)+\cdots+\sum_{\varphi} \sum_{\omega_{2}} a_{\tau \varphi}\left(l_{\omega_{n \tau-1}}^{(\tau)} h_{\omega_{n \tau-1}}^{(\tau)} \mid l_{\omega_{2}}^{(\varphi)} h_{\omega_{2}}^{(\varphi)} ; V_{\mathrm{E}}^{\mathrm{BT}}\right) \times \\
& \times y_{\varphi}\left(0, l_{2} ; l_{\omega_{2}}^{(\varphi)} h_{\omega_{2}}^{(\varphi)} ; V_{\mathrm{E}}^{\mathrm{BT}}\right)+\sum_{\psi} \sum_{\omega_{1}} a_{\tau \psi}\left(l_{\omega_{n_{\tau}-1}}^{(\tau)} h_{\omega_{n_{\tau}-1}}^{(\tau)} \mid l_{\omega_{1}}^{(\psi)} h_{\omega_{1}}^{(\psi)} ; V_{\mathrm{E}}^{\mathrm{BT}}\right) \times \\
& \times\left[y_{\psi}\left(0, l_{1} ; l_{\omega_{1}}^{(\psi)} h_{\omega_{1}}^{(\psi)} ; V_{\mathrm{E}}^{\mathrm{BT}}\right)+x_{\psi}\left(0, l_{1} ; l_{\omega_{1}}^{(\psi)} h_{\omega_{1}}^{(\psi)} ; V_{\mathrm{E}}^{\mathrm{BT}}\right)\right]
\end{aligned}
$$

The coefficients $a_{\tau \chi}\left(l_{\omega_{1+j}}^{(\tau)} h_{\omega_{i+j}}^{(\tau)} \mid l_{\omega_{\tau}}^{(\chi)} h_{\omega_{1}}^{(\chi)} ; V_{\mathrm{E}}^{\mathrm{BT}}\right)$ can be easily and directly determined from the geometry of the lattice site clusters.

The lattice site clusters $l_{\omega_{i}}^{(r)} h_{\omega_{i}}^{(r)}$ on the correlation or super correlation lattices of the basic compound lattice or the pseudo lattices can be occupied by particles of different species in various ways. Each state may be denoted by an index $K$. The event that the lattice site cluster $l_{\omega_{i}}^{(\tau)} h_{\omega_{i}}^{(\tau)}$ is occupied or is not occupied in the state $K$ may be characterized by an occupation variable $P\left(l_{\omega_{i}}^{(\tau)} h_{\omega_{i}}^{(\tau)}, K ; V_{\mathrm{E}}^{\mathrm{BT}}\right)$ with

$$
P\left(l_{\omega_{i}}^{(\tau)} h_{\omega_{i}}^{(\tau)}, K ; V_{\mathrm{E}}^{\mathrm{BT}}\right)=\left\{\begin{array}{l}
1 \text { when occupied in the way } K \\
0 \text { when not occupied in the way } K
\end{array}\right.
$$

The (ensemble) mean value $\left\langle P\left(l_{\omega_{i}}^{(\tau)} h_{\omega_{1}}^{(\tau)}, K ; V_{E}^{\mathrm{BT}}\right)\right\rangle$ of this variable gives the ratio of the number of lattice site clusters occupied in the way $K$ to the whole number of lattice site clusters $l_{\omega_{i}}^{(\tau)} h_{\omega_{i}}^{(\tau)}$ that is the statistical probability of a corresponding occupation $K$. Among the different states of occupation, there are those 
which are generated from one another by symmetry operations and which have therefore the same probability. The number of these states is generally considered by a multiplicity coefficient $j_{r}\left(l_{\omega_{j}}^{(\tau)} h_{\omega_{i}}^{(\tau)}, K ; V_{\mathrm{E}}^{\mathrm{BD}}\right)$. The lattice site cluster probabilities are not independent in general but they must fulfill the following consistency relations :

$$
\rho_{0}\left(\mathrm{n}, V_{\mathrm{E}}^{\mathrm{BT}}\right)=1-\sum_{r=1}^{N} \rho_{r}\left(\mathrm{n}, V_{\mathrm{E}}^{\mathrm{BT}}\right), \sum_{K}\left\langle P\left(l_{\omega_{i}}^{(\tau)} h_{\omega_{i}}^{(\tau)}, K ; V_{\mathrm{E}}^{\mathrm{BT}}\right)\right\rangle j_{\tau}\left(l_{\omega_{i}}^{(\tau)} h_{\omega_{i}}^{(\tau)}, K ; V_{\mathrm{E}}^{\mathrm{BT}}\right)=1
$$

normalization

$$
\sum_{K}^{\prime}\left\langle P\left(I_{\omega_{i}}^{(\tau)} h_{\omega_{i}}^{(\tau)}, K ; V_{\mathrm{E}}^{\mathrm{BT}}\right)\right\rangle=\rho_{r}\left(\mathbf{n}, V_{\mathrm{E}}^{\mathrm{BT}}\right)
$$

single lattice site consistency and more general

$$
\sum_{K}^{\prime}\left\langle P\left(l_{\omega_{i}}^{(\tau)} h_{\omega_{i}}^{(\tau)}, K ; V_{\mathrm{E}}^{\mathrm{BT}}\right)\right\rangle=\left\langle P\left(l_{\omega_{j}}^{(\chi)} h_{\omega_{j}}^{(\chi)}, H ; V_{\mathrm{E}}^{\mathrm{BT}}\right)\right\rangle
$$

cluster consistency.

The restrictive (primed) sums in (9) and (10) over occupation states of fixed lattice site clusters which contain a single lattice site of sublattice $\mathbf{n}$ or a lattice site cluster $l_{\omega_{j}}^{(x)} h_{\omega_{i}}^{(x)}$ respectively extend over all those states, where the single lattice site of sublattice $\mathbf{n}$ is occupied by one particle of species $\mathbf{r}$ or the subcluster $l_{\omega}^{(x)} h_{\omega}^{(x)}$ is occupied in the way $H$ respectively. $\rho_{0}\left(n, V_{E}^{B T}\right)$ in $(8)$ is the hole density. As the normalization-consistency relations (8)-(10) are linear all probabilities can be represented by linear combinations of a set of independent lattice site cluster probabilities $\left\langle P\left(l_{\omega_{j}}^{(\lambda)} h_{\omega_{j}}^{(\lambda)}, K^{\prime} ; V_{\mathrm{E}}^{\mathrm{BT}}\right)\right\rangle_{\mathrm{u}}$ and the sublattice densities $\rho_{s}\left(\mathbf{m}, V_{\mathrm{E}}^{\mathrm{BT}}\right)$ :

$$
\begin{aligned}
&\left\langleP \left( l_{\omega_{i}}^{(\tau)} h_{\omega_{i}}^{(\tau)}, K ;\right.\right.\left.\left.V_{\mathrm{E}}^{\mathrm{BT}}\right)\right\rangle=\sum_{\lambda=1}^{\sigma} \sum_{j=1}^{n_{\lambda}-1} \sum_{\omega_{j}} \sum_{K^{\prime}} q\left(l_{\omega_{i}}^{(\tau)} h_{\omega_{i}}^{(\tau)}, K \mid l_{\omega_{j}}^{(\lambda)} h_{\omega_{i}}^{(\lambda)}, K^{\prime} ; V_{\mathrm{E}}^{\mathrm{BT}}\right) \times \\
& \times\left\langle P\left(l_{\omega_{j}}^{(\lambda)} h_{\omega_{j}}^{(\lambda)}, K^{\prime} ; V_{\mathrm{E}}^{\mathrm{BT}}\right)\right\rangle_{\mathrm{u}}+\sum_{\mathbf{m}}\left(\sum_{s=1}^{N} q\left(l_{\omega_{i}}^{(\tau)} h_{\omega_{i}}^{(\tau)}, K \mid s, m ; V_{\mathrm{E}}^{\mathrm{BT}}\right) \rho_{s}\left(\mathbf{m}, V_{\mathrm{E}}^{\mathrm{BT}}\right)+\right. \\
&\left.\quad+q\left(l_{\omega_{i}}^{(\tau)} h_{\omega_{i}}^{(\tau)}, K \mid 0, \mathbf{m} ; V_{\mathrm{E}}^{\mathrm{BT}}\right)\left(1-\sum_{s=1}^{\mathrm{B}} \boldsymbol{\rho}_{s}\left(\mathbf{m}, V_{\mathrm{E}}^{\mathrm{BT}}\right)\right)\right) .
\end{aligned}
$$

After the preliminaries, presented above, $W_{\text {conf }}\left(V_{\mathrm{E}}^{\mathrm{BT}}\right)$ and $\left\langle\varepsilon\left(V_{\mathrm{E}}^{\mathrm{BT}}\right)\right\rangle$ can be calculated in a very similar way as has been done for homogeneous phase systems. The evaluation of $W_{\text {conf }}\left(V_{\mathrm{E}}^{\mathrm{BT}}\right)$ can be reduced thereby to the counting of certain numbers of configurations $Q\left(0, l_{h},-, l_{i} ;\left\{\left\langle P\left(l_{\omega}^{(\rho)} h_{\omega}^{(\rho)}, K ; V_{\mathrm{E}}^{\mathrm{BT}}\right)\right\rangle\right\}\right)$ on pseudo lattices $p\left(0, l_{h},-, l_{i} ; V_{\mathrm{E}}^{\mathrm{BT}}\right)$ with a complete set of fixed cluster probabilities $\left\{\left\langle\boldsymbol{P}\left(\boldsymbol{l}_{\omega_{\mathrm{s}}}^{(\rho)} \boldsymbol{h}_{\omega_{i}}^{(\rho)}, K ; V_{\mathrm{E}}^{\mathrm{BT}}\right)\right\rangle\right\}$ which are defined on corresponding super correlation lattices $\rho$ with clusters belonging to definite positions $j$ in the sequences of overlap figures. The crucial approximation in this procedure are the combinatorial ansätze

$$
\Gamma\left(0, l_{r},-, l_{m} \mid 0, l_{r},-, l_{m} ; l_{n} ; V_{\mathrm{E}}^{\mathrm{BT}}\right)=\left[\Gamma\left(0, l_{r},-, l_{m} \mid 0, l_{r},-, l_{m} ; l_{n+1} ; V_{\mathrm{E}}^{\mathrm{BT}}\right)\right]^{\alpha\left(0, l_{r}-l_{m} ; l_{n}\right)}
$$

between the following ratios of configuration numbers :

$$
\begin{gathered}
\frac{Q\left(0, l_{r},-, l_{m} ;\left\{\left\langle P\left(l_{\omega_{n}}^{(\rho)} h_{\omega_{n}}^{(\rho)}, K ; V_{\mathrm{E}}^{\mathrm{BT}}\right)\right\rangle\right\}\right)}{Q\left(0, l_{r},-, l_{m}, l_{n} ;\left\{\left\langle P\left(l_{\omega_{n}}^{\left(\rho_{n}\right)} h_{\omega_{n}}^{(\rho)}, K ; V_{\mathrm{E}}^{\mathrm{BT}}\right)\right\rangle\right\}\right)}=\Gamma\left(0, l_{\mathrm{r}},-, l_{\mathrm{m}} \mid 0, l_{\mathrm{r}},-, l_{\mathrm{m}} ; l_{n} ; V_{\mathrm{E}}^{\mathrm{BT}}\right), \\
\frac{Q\left(0, l_{r},-, l_{m} ;\left\{\left\langle P\left(l_{\omega_{n+1}}^{(\rho)} h_{\omega_{n+1}}^{(\rho)}, K ; V_{\mathrm{E}}^{\mathrm{BD}}\right)\right\rangle\right\}\right)}{Q\left(0, l_{r},-, l_{m}, l_{n} ;\left\{\left\langle P\left(l_{\omega_{n+1}}^{(\rho)} h_{\omega_{n+1}}^{(\rho)}, K ; V_{\mathrm{E}}^{\mathrm{BT}}\right)\right\rangle\right\}\right)}=\Gamma\left(0, l_{r},-, l_{m} \mid 0, l_{r},-, l_{m} ; l_{n+1} ; V_{\mathrm{E}}^{\mathrm{BT}}\right) .
\end{gathered}
$$

The exponents $\alpha\left(0, l_{r},-, l_{m} ; l_{n}\right)$ in (12) can be generally assumed as arbitrary slowly varying functions of the sublattice densities and the cluster probabilities. The most familiar approximation is [8-10]

$$
\alpha\left(0, l_{r},-, l_{m} ; l_{n} ; V_{\mathrm{E}}^{\mathrm{BT}}\right) \equiv 1 .
$$

On the other hand, some investigations of simple lattice models by these approximation methods have shown that values of $\alpha\left(0, l_{r},-, l_{m} ; l_{n} ; V_{\mathrm{E}}^{\mathrm{B} T}\right)$ which are different from (15) can be more appropriate in certain regions of the density, whereby more appropriate phase diagrams are delivered [12-13]. The equations (12)-(14) allow an elimination of the configuration numbers

$$
Q\left(0, l_{r},-, l_{m} ;\left\{\left\langle P\left(l_{\omega_{n}}^{(\rho)} h_{\omega_{n}}^{(\rho)}, K ; V_{\mathrm{E}}^{\mathrm{BT}}\right)\right\rangle\right\}\right)
$$


with $m \neq n$ and an expression of the number $Q\left(0 ;\left\{\left\langle P\left(l_{\omega_{1}}^{(\rho)} h_{\omega}^{(\rho)}, K ; V_{\mathrm{E}}^{\mathrm{BT}}\right)\right\rangle\right\}\right)$ as a quotient of multiple products with powers of the configuration numbers $Q\left(0, l_{r},-, l_{n} ;\left\{\left\langle P\left(l_{\omega_{n}}^{(\rho)} h_{\omega_{n}}^{(\rho)}, K ; V_{E}^{\mathrm{B} T}\right)\right\rangle\right\}\right)$ as factors. These configuration numbers however can be easily calculated by the combinatorial formula

$$
\begin{aligned}
Q\left(0, l_{r},-, l_{n} ;\left\{\left\langle P\left(l_{\omega_{n}}^{(\rho)} h_{\omega_{n}}^{(\rho)}, K ; V_{\mathrm{E}}^{\mathrm{BT}}\right)\right\rangle\right\}\right)= \\
=\prod_{\rho=1}^{\varphi} \prod_{\omega_{n}} \frac{\times\left[x_{\rho}\left(0, l_{r},-, l_{n} ; l_{\omega_{n}}^{(\rho)} h_{\omega_{n}}^{(\rho)} ; V_{\mathrm{E}}^{\mathrm{BT}}\right) V\right] !}{\left.\prod_{K}\left\{\left[P\left(l_{\omega_{n}}^{(\rho)} h_{\omega_{n}}^{(\rho)}, K ; V_{\mathrm{E}}^{\mathrm{BT}}\right)\right\rangle x_{\rho}\left(0, l_{r},-, l_{n} ; l_{\omega_{n}}^{(\rho)} h_{\omega_{n}}^{(\rho)} ; V_{\mathrm{E}}^{\mathrm{BT}}\right) V\right] !\right\} j_{\rho}\left(l_{\omega_{n}}^{(\rho)} h_{\omega_{n}}^{(\rho)}, K ; V_{\mathrm{E}}^{\mathrm{BT}}\right)}
\end{aligned}
$$

When defining the combinatorial coefficients

$$
z_{\tau}\left(0, l_{1},-, l_{i} ; l_{\omega_{i}}^{(\tau)} h_{\omega_{i}}^{(\tau)} ; V_{\mathrm{E}}^{\mathrm{BT}}\right)=\sum_{\left\{k_{1},-, k_{j}\right\}} f_{k_{1},-, k_{j}}^{(\tau)}\left(\left\{\alpha\left(0, l_{r},-, l_{m} ; l_{n}\right)\right\}\right) x_{r}\left(0, l_{k_{i}},-, l_{k_{j}}, l_{i} ; l_{\omega_{i}}^{(\tau)} h_{\omega_{i}}^{(\tau)} ; V_{\mathrm{E}}^{\mathrm{B})}\right),
$$

on the correlation lattices, whereby the sum extends over all combinations $k_{1}, k_{2},-, k_{j}$ with $1 \leqslant k_{1}<k_{2}<\cdots<k_{j} \leqslant i-1$ and the coefficients $f_{k_{1},-, k_{j}}^{(r)}\left(\left\{\alpha\left(0, l_{n},-, l_{m} ; l_{n}\right)\right\}\right)$ are algebraic multilinear forms of sets $\left\{\alpha\left(0, l_{r},-, l_{m} ; l_{n}\right)\right\}$, then the approximation

$$
W_{\text {conf }}\left(V_{\mathrm{E}}^{\mathrm{BT}}\right)=Q\left(0 ;\left\{\left\langle P\left(l_{\omega_{1}}^{(\rho)} h_{\omega_{1}}^{(\rho)}, K ; V_{\mathrm{E}}^{\mathrm{BT}}\right)\right\rangle\right\}\right)
$$

leads with the aid of Stirling's formula to the entropy formula :

$$
\begin{aligned}
\frac{1}{V} \ln \left[W_{\mathrm{conf}}\left(V_{\mathrm{E}}^{\mathrm{BT}}\right)\right]=\sum_{\tau=1}^{\sigma} \sum_{i=1}^{n_{\tau}} \sum_{\omega_{i}} z_{\tau}\left(0, l_{i},-, l_{i} ; l_{\omega_{i}}^{(\tau)} h_{\omega_{i}}^{(\tau)} ; V_{\mathrm{E}}^{\mathrm{BT}}\right) \times \\
\quad \times \sum_{K} j_{\tau}\left(l_{\omega_{i}}^{(\tau)} h_{\omega_{i}}^{(\tau)}, K ; V_{\mathrm{E}}^{\mathrm{BT}}\right)\left\langle P\left(l_{\omega_{i}}^{(\tau)} h_{\omega_{i}}^{(\tau)}, K ; V_{\mathrm{E}}^{\mathrm{BT}}\right)\right\rangle \ln \left[\left\langle P\left(l_{\omega_{i}}^{(\tau)} h_{\omega_{i}}^{(\tau)}, K ; V_{\mathrm{E}}^{\mathrm{BT}}\right)\right\rangle\right] .
\end{aligned}
$$

In formula (19) the cluster probabilities must be expressed by the independent cluster probabilities and the sublattice densities from (11). The consideration of configuration probabilities on the correlation lattices as in (19) instead of those on super correlation lattices as in (16) is correct, because the product over the super correlation lattice index $\rho$ in (16) factorizes in terms defined for the correlation lattices $\tau$ in the same way. This means that

$$
\begin{gathered}
z_{\rho}\left(0, l_{1},-, l_{i} ; l_{\omega_{i}}^{(\rho)} h_{\omega_{i}}^{(\rho)} ; V_{\mathrm{E}}^{\mathrm{RT}}\right)=m_{\rho} z_{\tau}\left(0, l_{1},-, l_{i} ; l_{\omega_{i}}^{(\tau)} h_{\omega_{i}}^{(\tau)} ; V_{\mathrm{E}}^{\mathrm{BT}}\right), \\
x_{\rho}\left(0, l_{k_{1}},-, l_{k_{i}}, l_{i} ; l_{\omega_{1}}^{(\rho)} h_{\omega_{i}}^{(\rho)} ; V_{\mathrm{E}}^{\mathrm{BT}}\right)=m_{\rho} x_{\tau}\left(0, l_{k_{1}},-, l_{k_{1}}, l_{i} ; l_{\omega_{i}}^{(\tau)} h_{\omega_{i}}^{(\tau)} ; V_{\mathrm{E}}^{\mathrm{BT}}\right), \\
f_{k_{1},-, k_{j}}^{(\rho)}=f_{k_{1},-, k_{i}}^{(\tau)} \\
\left\langle P\left(l_{\omega_{i}}^{(\rho)} h_{\omega_{i}}^{(\rho)}, K ; V_{\mathrm{E}}^{\mathrm{BT}}\right)\right\rangle=\left\langle P\left(l_{\omega_{i}}^{(\tau)} h_{\omega_{i}}^{(\tau)}, K ; V_{\mathrm{E}}^{\mathrm{BT}}\right)\right\rangle
\end{gathered}
$$

is valid.

The mean configurational energy per lattice site $\left\langle\varepsilon\left(V_{\mathrm{E}}^{\mathrm{BT}}\right)\right\rangle$ can be also expressed as a sum over cluster contributions like the entropy in (19). The configurational energy $\varepsilon_{\tau}\left(l_{\omega_{i}}^{(\tau)} h_{\omega_{i}}^{(\tau)}, K ; V_{E}^{\mathrm{BT}}\right)$ of one lattice site cluster $l_{\omega_{i}}^{(\tau)} h_{\omega_{i}}^{(\tau)}$ occupied in the way $K$ is defined by the restricted sum

$$
\boldsymbol{\varepsilon}_{\tau}\left(l_{\omega_{i}}^{(\tau)} \boldsymbol{h}_{\omega_{i}}^{(\tau)}, K ; V_{\mathrm{E}}^{\mathrm{BT}}\right)=-\sum_{(r, i, s, j)}^{\prime} \boldsymbol{v}_{r s}\left(\left|\mathbf{r}_{i}-\mathbf{r}_{j}\right|\right)
$$

which extends over all interacting pairs of particles in the $K$-occupied cluster $l_{\omega_{t}}^{(\tau)} h_{\omega_{i}}^{(\tau)}$. When all states of occupation are admitted, the mean configurational cluster energy per lattice site of the cluster $l_{\omega_{i}}^{(\tau)} h_{\omega_{i}}^{(\tau)}$ is represented by the term

$$
\sum_{K} \varepsilon_{\tau}\left(l_{\omega_{i}}^{(\tau)} h_{\omega_{i}}^{(\tau)}, K ; V_{\mathrm{E}}^{\mathrm{BT}}\right) j_{\tau}\left(l_{\omega_{i}}^{(\tau)} h_{\omega_{i}}^{(\tau)}, K ; V_{\mathrm{E}}^{\mathrm{BT}}\right)\left\langle P\left(l_{\omega_{i}}^{(\tau)} h_{\omega_{i}}^{(\tau)}, K ; V_{\mathrm{E}}^{\mathrm{BT}}\right)\right\rangle x_{\tau}\left(0, l_{i} ; l_{\omega_{i}}^{(\tau)} h_{\omega_{i}}^{(\tau)} ; V_{\mathrm{E}}^{\mathrm{BT}}\right)
$$

The sum $\sum_{\tau=1}^{\sigma} \sum_{i=1}^{n_{i}-1} \sum_{\omega_{i}}$ over all cluster contributions of terms like (22), however, does not reproduce the correct cluster part of the mean configurational energy on account of an overcounting of the contributions of the overlapping parts of the clusters. On the other hand, this overcounting can be avoided by using in (22) the correction terms $y_{\tau}\left(0, l_{\tau} ; l_{\omega_{1}}^{(\tau)} h_{\omega_{i}}^{(\tau)} ; V_{\mathrm{E}}^{\mathrm{BT}}\right.$ ) (defined by (7)) instead of the cluster distribution numbers $x_{\tau}\left(0, l_{i} ; l_{\omega_{\tau}}^{(\tau)} h_{\omega_{i}}^{(\tau)} ; V_{\mathrm{E}}^{\mathrm{BT}}\right)$. In this way, the correct formula for the cluster part of the mean configurational energy per lattice site is given by: 


$$
\begin{aligned}
\sum_{\tau=1}^{\sigma} \sum_{i=1}^{n_{\tau}-1} \sum_{\omega_{i}}\left[y_{\tau}\left(0, l_{i} ; l_{\omega_{i}}^{(\tau)} h_{\omega_{i}}^{(\tau)} ; V_{\mathrm{E}}^{\mathrm{BT}}\right)\right. & \left.+\delta_{1 i} x_{\tau}\left(0, l_{2} ; l_{\omega_{1}}^{(\tau)} h_{\omega_{1}}^{(\tau)} ; V_{\mathrm{E}}^{\mathrm{BT}}\right)\right] \times \\
& \times \sum_{K} \varepsilon_{\tau}\left(l_{\omega_{i}}^{(\tau)} h_{\omega_{1}}^{(\tau)}, K ; V_{\mathrm{E}}^{\mathrm{BT}}\right) j_{\tau}\left(l_{\omega_{i}}^{(\tau)} h_{\omega_{1}}^{(\tau)}, K ; V_{\mathrm{E}}^{\mathrm{BT}}\right)\left\langle P\left(l_{\omega_{i}}^{(\tau)} h_{\omega_{i}}^{(\tau)}, K ; V_{\mathrm{E}}^{\mathrm{BT}}\right)\right\rangle,
\end{aligned}
$$

where $\delta_{1 i}$ is the Kronecker symbol.

Apart from (23), there is a mean-field contribution to $\left\langle\varepsilon\left(V_{\mathrm{E}}^{\mathrm{BT}}\right)\right\rangle$ which arises from the uncorrelated lattice sites with distances lying in the interaction zone $K=\Omega$. This contribution is apart from the sign completely equivalent with (4) with the exception that the summations of the interaction terms $w_{r s}\left(\mathbf{m}, \mathbf{n}, ; V_{\mathrm{E}}^{\mathrm{BT}}\right)$ as defined in (5) are restricted to pairs of lattice sites at a distance lying in the zone $K=\Omega$.

The complete pressure-density function in this most general case has the form

$$
\begin{aligned}
p v & =\left\{-\sum_{\tau=1}^{\sigma} \sum_{i=1}^{n_{\tau}-1} \sum_{\omega_{i}}\left[y_{\tau}\left(0, l_{i} ; l_{\omega_{i}}^{(\tau)} h_{\omega_{i}}^{(\tau)} ; V_{\mathrm{E}}^{\mathrm{BT}}\right)+\right.\right. \\
& \left.+\delta_{1 i} x_{\tau}\left(0, l_{i} ; l_{\omega_{i}}^{(\tau)} h_{\omega_{i}}^{(\tau)} ; V_{\mathrm{E}}^{\mathrm{BT}}\right)\right] \sum_{K} \varepsilon_{\tau}\left(l_{\omega_{i}}^{(\tau)} h_{\omega_{i}}^{(\tau)}, K ; V_{\mathrm{E}}^{\mathrm{BT}}\right) \times \\
& \times j_{\tau}\left(l_{\omega_{i}}^{(\tau)} h_{\omega_{i}}^{(\tau)}, K ; V_{\mathrm{E}}^{\mathrm{BT}}\right)\left\langle P\left(l_{\omega_{i}}^{(\tau)} h_{\omega_{\tau}}^{(\tau)}, K ; V_{\mathrm{E}}^{\mathrm{BT}}\right)\right\rangle+ \\
& +\frac{1}{2} \sum_{r, s=1}^{N} \sum_{\mathbf{n}}^{\alpha(V)} \sum_{\mathbf{m}}^{\alpha(\mathrm{V})} g /\left(\mathbf{n}, V_{\mathrm{E}}^{\mathrm{BT}}\right) w_{r s}\left(\mathbf{n}, \mathbf{m} ; V_{\mathrm{E}}^{\mathrm{BT}}\right) \\
& \left.\times \rho_{r}\left(\mathbf{n}, V_{\mathrm{E}}^{\mathrm{BT}}\right) \rho_{s}\left(\mathbf{m}, V_{\mathrm{E}}^{\mathrm{BT}}\right)+\sum_{s=1}^{N}\left[\sum_{\mathbf{n}} g\left(\mathbf{n}, V_{\mathrm{E}}^{\mathrm{BT}}\right) \rho_{s}\left(\mathbf{n}, V_{\mathrm{E}}^{\mathrm{BT}}\right)\right] \mu_{s}\right\}+ \\
& +\frac{1}{\beta}\left\{\sum_{\tau=1}^{\sigma} \sum_{i=1}^{n_{\tau}} \sum_{\omega_{i}} z_{\tau}\left(0, l_{1},-, l_{i} ; l_{\omega_{i}}^{(\tau)} h_{\omega_{i}}^{(\tau)} ; V_{\mathrm{E}}^{\mathrm{BT}}\right) \times\right. \\
& \left.\times \sum_{K} j_{\tau}\left(l_{\omega_{i}}^{(\tau)} h_{\omega_{i}}^{(\tau)}, K ; V_{\mathrm{E}}^{\mathrm{BT}}\right)\left\langle P\left(l_{\omega_{i}}^{(\tau)} h_{\omega_{i}}^{(\tau)}, K ; V_{\mathrm{E}}^{\mathrm{BT}}\right)\right\rangle \ln \left[\left\langle P\left(l_{\omega_{i}}^{(\tau)} h_{\omega_{i}}^{(\tau)}, K ; V_{\mathrm{E}}^{\mathrm{BT}}\right)\right\rangle\right]\right\},
\end{aligned}
$$

where by the cluster probabilities $\left\langle P\left(l_{\omega_{i}}^{(\tau)} h_{\omega_{i}}^{(\tau)}, K ; V_{\mathrm{E}}^{\mathrm{BT}}\right)\right\rangle$ must be expressed via (11) by the independent cluster probabilities $\left\langle P\left(l_{\omega_{r}}^{(\tau)} h_{\omega}^{(\tau)}, K ; V_{\mathrm{E}}^{\mathrm{BT}}\right)\right\rangle_{\mathrm{u}}$ and the sublattice densities $\rho_{r}\left(\mathbf{n}, V_{\mathrm{E}}^{\mathrm{BT}}\right)$.

The pressure function has extremum properties with respect to the macroscopic variables $\left\langle P\left(l_{\omega_{i}}^{(\tau)} h_{\omega_{i}}^{(\tau)}, K ; V_{\mathrm{E}}^{\mathrm{BT}}\right)\right\rangle_{\mathrm{u}}$ and $\rho_{r}\left(\mathrm{n}, V_{\mathrm{E}}^{\mathrm{BT}}\right)$, that means the partial derivatives with respect to these variables must vanish :

$$
\frac{\partial p}{\partial\left\langle P\left(l_{\omega_{i}}^{(\tau)} h_{\omega_{i}}^{(\tau)}, K ; V_{\mathrm{E}}^{\mathrm{BT}}\right)\right\rangle_{\mathrm{u}}}=0, \quad \frac{\partial p}{\partial \rho_{r}\left(\mathrm{n}, V_{\mathrm{E}}^{\mathrm{BT}}\right)}=0 .
$$

Equations (25) form a complete closed-form coupled set of transcendental state equations which deliver the functions $p\left(\mu_{1},-, \mu_{N}\right)$ and $\rho_{r}\left(\mathbf{n}, V_{\mathrm{E}}^{\mathrm{BT}} ; \mu_{1},-, \mu_{N}\right)$ as solutions. A practical solution of (25) with an afterward calculation of (24) is however very difficult and only tractable by computer procedures. The function $p\left(\mu_{1},-, \mu_{N}\right)$ is an ambiguous function over parts of the $\mu_{1},-, \mu_{N}$ space in the sense that for certain sets $\mu_{1},-, \mu_{N}$ there are several different values of $p$ belonging to fluid states, or to crystalline states of different types $\mathrm{BT}$, or different volumes $V_{\mathrm{E}}^{\mathrm{BT}}$ of the elementary cell, or different density structures of the particle distribution. From these different values, however, only that with maximum pressure $p$ is of physical interest. This value is uniquely adjoined to a fluid- or crystalline structure of fixed type BT, fixed volume $V_{E}^{\mathrm{BT}}$ and fixed density distribution and characterizes thermodynamical equilibrium. For this reason, of course, the lattice constant of a crystalline structure is also determined. A real determination, however, is only possible with the aid of a computer.

The general formulas (24)-(25) include also the special case of the mean-field approximation [7]. One needs only neglect the cluster contribution in (24) and replace $W_{\text {conf }}$ by the expression (3).

An extension of the hard-core interaction beyond the simple prohibition of multiple occupancy of a single lattice site is also possible. In this case, the cluster probabilities of those clusters $l_{\omega_{*}}^{(\tau)} h_{\omega_{i}}^{(\tau)}$ which can be occupied on account of the hard-core interaction by maximal one particle obey instead of (8)-(10) the following hard-core consistency relations :

$$
\begin{gathered}
\left\langle P\left(l_{\omega_{i}}^{(\tau)} h_{\omega_{i}}^{(\tau)}, K\left(\mathbf{n}_{1},-, \mathbf{n}_{j}\right) ; V_{\mathrm{E}}^{\mathrm{BT}}\right)\right\rangle=0, \\
\rho_{r}\left(\mathbf{n}, V_{\mathrm{E}}^{\mathrm{BT}}\right)=\left\langle P\left(l_{\omega_{i}}^{(\tau)} h_{\omega_{i}}^{(\tau)}, K(r, \mathbf{n}) ; V_{\mathrm{E}}^{\mathrm{BT}}\right)\right\rangle, \\
\left\langle P\left(l_{\omega_{i}}^{(\tau)} h_{\omega_{i}}^{(\tau)}, K(0) ; V_{\mathrm{E}}^{\mathrm{BT}}\right)\right\rangle=1-\sum_{\mathbf{m}} f\left(\mathbf{m}, l_{\omega_{i}}^{(\tau)} h_{\omega_{i}}^{(\tau)}\right) \sum_{s=1}^{N} \rho_{s}\left(\mathbf{m}, V_{\mathrm{E}}^{\mathrm{BT}}\right) .
\end{gathered}
$$


$K\left(\mathbf{n}_{1},-, \mathbf{n}_{j}\right)$ in (26) denotes thereby a state of an occupation of the lattice site cluster $l_{\omega_{1}}^{(\tau)} h_{\omega_{i}}^{(\tau)}$ with $j$ particles of arbitrary species $\left(2 \leq j \leq l_{\omega_{i}}^{(r)}\right)$ each put on a site of the sublattices $\mathbf{n}_{1}, \mathbf{n}_{2},-, \mathbf{n}_{j}$, where some $\mathbf{n}_{h}$ may coincide. $K(r, \mathbf{n})$ in $(27)$ denotes a state of an occupation of $l_{\omega \rightarrow}^{(\tau)} h_{\omega_{i}}^{(\tau)}$ with one particle of species $r$ put on a site of sublattice $\mathrm{n} . K(0)$ in (28) denotes the empty state of $l_{\omega_{l}}^{(\tau)} h_{\omega_{i}}^{(\tau)}$ and $f\left(\mathbf{m}, l_{\omega_{i}}^{(\tau)} h_{\omega_{i}}^{(\tau)}\right)$ denotes the number of lattice sites of sublattice $\mathrm{m}$ belonging to $l_{\omega_{i}}^{(\tau)} h_{\omega_{i}}^{(\tau)}$. For the other clusters which can be occupied by more than one particle (8)-(10) remain valid, but in the sums, the contributions of the clusters with probabilities fulfilling (26) must be omitted then. Further, the contributions to the mean configurational energy term in (24) of the lattice site clusters fulfilling (26)-(28) must be dropped. On the other hand, the cluster probabilities of these clusters contribute in every case to the combinatorial factor term in (24). A considerable extension of the range of the hard-core interaction can be now chosen in the way that all distances lying in the interaction zones $K=1,-, r(r \leq \Omega-1)$ are smaller than the hard-core diameter. An especial simple case is obtained, if $r=\Omega-1$ is chosen. In this case, only the mean-field contribution to the $\left\langle\varepsilon\left(V_{\mathrm{E}}^{\mathrm{BT}}\right)\right\rangle$ term in $(24)$ needs to be considered.

Calculations for some examples have shown that these simple approximations are able to deliver reasonable results for phase systems which are not too complex [11-14]. For this reason, a generalization of the lattice-model theory of multiphase systems, as represented by formulas (24), (25), appears to be reasonable.

\section{References}

[1] RunNels, L. K., in, Phase Transitions and Critical Phaenomena (Academic Press, New York), 1972, Vol. II, ed. by C. Domb and M. S. Green, S. 305.

[2] Burley, D. M., in, Phase Transitions and Critical Phaenomena (Academic Press, New York), 1972, Vol. II, ed. by C. Domb and M. S. Green, S. 329.

[3] Kaye, R. D. and Burley, D. M., J. Phys. A 7 (1974) 843.

[4] Kaye, R. D. and Burley, D. M., J. Phys. A 7 (1974) 1303.

[5] Horiguchi, T. and Tanaka, T., Phys. Rev. A 7 (1973) 1723.
[6] Schouten, J. A., Ten Seldam, C. A. and Trappeniers, N. J., Physica 73 (1974) 556.

[7] Neumann, H. P., Phys. Rev. B 14 (1976) 4146.

[8] Humans, J. and DE BOER, J., Physica 21 (1955) 471

[9] Humans, J. and DE BoER, J., Physica 21 (1955) 485.

[10] HIJMans, J. and DE BoER, J., Physica 21 (1955) 499.

[11] NeumanN, H. P., Z. Naturforsch. 29a (1974) 65.

[12] NeumanN, H. P., J. Stat. Phys. 13 (1975) 173.

[13] Neumann, H. P., Phys. Rev. A 11 (1975) 1043.

[14] Neumann, H.P., Phys. Rev. A 12 (1975) 1591. 